\title{
Islet Transplantation under the Kidney Capsule Fully Corrects the Impaired Skeletal Muscle Glucose Transport System of Streptozocin Diabetic Rats
}

\author{
Raffaele Napoli, Alberto M. Davalli, Michael F. Hirshman, Raimund Weitgasser, Gordon C. Weir, and Edward S. Horton
}

Research Division, Joslin Diabetes Center, and Harvard Medical School, Boston, Massachusetts 02215

\begin{abstract}
Chronic insulin therapy improves but does not restore impaired insulin-mediated muscle glucose uptake in human diabetes or muscle glucose uptake, transport, and transporter translocation in streptozocin diabetic rats. To determine whether this inability is due to inadequate insulin replacement, we studied fasted streptozocin-induced diabetic Lewis rats either untreated or after islet transplantation under the kidney capsule. Plasma glucose was increased in untreated diabetics and normalized by the islet transplantation $(110 \pm 5,452 \pm 9$, and $102 \pm 3 \mathrm{mg} / \mathrm{dl}$ in controls, untreated diabetics, and transplanted diabetics, respectively). Plasma membrane and intracellular microsomal membrane vesicles were prepared from hindlimb skeletal muscle of basal and maximally insulin-stimulated rats. Islet transplantation normalized plasma membrane carrier-mediated glucose transport $V_{\text {max }}$, plasma membrane glucose transporter content, and insulin-induced transporter translocation. There were no differences in transporter intrinsic activity $\left(V_{\max } /\right.$ $R_{\mathrm{o}}$ ) among the three groups. Microsomal membrane GLUT4 content was reduced by $30 \%$ in untreated diabetic rats and normal in transplanted diabetics, whereas the insulininduced changes in microsomal membrane GLUT4 content were quantitatively similar in the three groups. There were no differences in plasma membrane GLUT1 among the groups and between basal and insulin stimulated states. Microsomal membrane GLUT1 content was increased $60 \%$ in untreated diabetics and normalized by the transplantation. In conclusion, an adequate insulin delivery in the peripheral circulation, obtained by islet transplantation, fully restores the muscle glucose transport system to normal in streptozocin diabetic rats. (J. Clin. Invest. 1996. 97:1389-1397.) Key words: GLUT4 • GLUT1 • intrinsic activity • translocation • fractionation procedure
\end{abstract}

Part of this research was reported in abstract form at the Annual Meeting of the American Diabetes Association, held in Atlanta 1013 June 1995 (Diabetes. 1995. 44:143A).

Address correspondence to Raffaele Napoli, M.D., Medicina Interna, Facolta di Medicina, via Pansini, 5. 80131 Napoli, Italy. Phone: 81-746-3517; FAX: 81-746-3199; E-mail: SACCA@DS. CISED.UNINA.IT

Received for publication 18 October 1995 and accepted in revised form 4 December 1995.

J. Clin. Invest.

(c) The American Society for Clinical Investigation, Inc.

0021-9738/96/03/1389/09 \$2.00

Volume 97, Number 6, March 1996, 1389-1397

\section{Introduction}

During insulin stimulation, skeletal muscle glucose transport is mainly regulated by the GLUT4 protein (1-3), which is quantitatively the most relevant transporter in this tissue. GLUT4 translocates from an intracellular pool to the plasma membrane in response to acute insulin stimulation (4-7). Although insulin-stimulated muscle glucose uptake (8-12) and transport (13) are impaired in human diabetes, GLUT4 content is not reduced, either in the whole muscle (14-18) or in the plasma membrane (19). Therefore, the reduction of glucose transport that occurs in diabetic patients might be due to abnormalities in the cellular localization of glucose transporters or to a decrease in transporter intrinsic activity (20-27), as we have recently suggested (28), rather than to a reduction in muscle GLUT4 content. However, no data on transporter intrinsic activity in human diabetes are available. In both type I and type II diabetes mellitus, chronic insulin therapy improves but does not correct impaired insulin-mediated skeletal muscle glucose uptake $(8,29-31)$. This inability of insulin therapy to restore insulin-mediated glucose disposal is surprising in type I diabetic patients, in whom the primary defect is the insulin deficiency, but perhaps therapy was inadequate to normalize glucose metabolism truly.

Streptozocin diabetic rats are characterized by reduction of muscle glucose uptake $(32)$ and transport $(5,28)$ due to decreased GLUT4 content and insulin-induced transporter translocation $(5,28)$, and to impairment of transporter intrinsic activity (28). In this model of diabetes, similar to what happens in human diabetes, insulin therapy does not restore insulinmediated skeletal muscle glucose uptake (32) and insulin-stimulated glucose transport and transporter translocation to normal (33). However, when phlorizin is used instead of insulin to reduce plasma glucose, insulin-mediated skeletal muscle glucose uptake is normalized (32), and glucose transport and transporter translocation are improved (28). Thus, three hypotheses can be proposed to explain the inability of insulin therapy to restore glucose uptake, transport, and transporter translocation in human diabetes, as well as in the rat model of diabetes: (a) although insulin treatment reduces glucose level, the increase in plasma insulin induced by the chronic insulin therapy might exert per se a negative effect on insulin-stimulated muscle glucose uptake (34-36); (b) subcutaneous insulin therapy in rats cannot produce a completely normal glucose profile: variable high and low glucose values adversely influence glucose uptake by muscle; and/or $(c)$ the insulin therapy was not adequate in terms of route of administration (37), because insulin was not administered in the portal system, as occurs physiologically, but subcutaneously into the peripheral circulation.

The main objective of this study was to test the hypothesis that insulin delivery into the peripheral circulation is able to normalize fully the defects in the skeletal muscle glucose trans- 
port system in diabetes. For this purpose, we studied streptozocin diabetic rats treated with islet transplantation under the kidney capsule. An additional aim of this study was to verify the hypothesis that hypoinsulinemia, and not hyperglycemia, was the factor responsible for the decrease in transporter intrinsic activity reported in streptozocin diabetic rats studied in the basal nonfasted state (28). Therefore, we examined the skeletal muscle glucose transport system in animals in the fasting state. In the control rats, going from the fed to the fasting state reduces insulin levels, making them similar to the low insulin levels found in untreated diabetic animals.

To accomplish the above purposes, we prepared plasma membranes $(\mathrm{PM})^{1}$ and intracellular microsomal membranes (MsM) from hindlimb muscle of untreated diabetic and transplanted diabetic rats in the fasting state, either in the basal state or after maximal insulin stimulation. In both fractions, GLUT4 and GLUT1 content were measured. In PM vesicles, the maximal velocity of glucose transport $\left(V_{\max }\right)$ was measured, as were transporter number and average transporter intrinsic activity.

\section{Methods}

Animals and experimental design. Male inbred Lewis rats weighing 150-200 g were obtained from Taconic Farms (Germantown, NY) and used both as islet donors and recipients. All animals were housed at $23^{\circ} \mathrm{C}$ with a 12-h light/dark cycle and fed ad libitum for several days before the start of the study. The rats were then randomly divided into three different groups: controls $(n=10)$ were left untreated and studied 10 wk later; untreated diabetic rats $(n=10)$ were left untreated for $4 \mathrm{wk}$, and then they received a single intravenous injection of $65 \mathrm{mg} / \mathrm{Kg}$ body wt of streptozocin (Sigma Chemical Co., St. Louis, MO) and studied $6 \mathrm{wk}$ later; transplanted diabetic rats $(n=13)$ were made diabetic by a single intravenous injection of $65 \mathrm{mg} / \mathrm{Kg}$ body wt of streptozocin, 2-3 wk later underwent islet transplantation, and 8 wk later were studied. Rats were considered diabetic when the nonfasted blood glucose level was $>300 \mathrm{mg} / \mathrm{dl}$.

Islet isolation and transplantation. Islet isolation and transplantation were performed as previously described (38). Briefly, under anesthesia with sodium amobarbital (amytal sodium; Eli Lilly \& Co., Indianapolis, IN), a laparotomy was performed and the pancreas was exposed. The common bile duct was ligated and the ampulla of Vater was cannulated proximally with P-50 polyethylene tubing and injected with $10 \mathrm{ml}$ of M-199 medium (GIBCO BRL, Gaithersburg, MD) containing $1.5 \mathrm{mg}$ of collagenase (type $\mathrm{P}$, Boehringer Mannheim, Mannheim, Germany) per milliliter. The pancreas was dissected from the surrounding tissues, removed, and incubated in a stationary bath for $15-18 \mathrm{~min}$ at $30^{\circ} \mathrm{C}$. After incubation, the digested tissue was washed with M-199 medium containing 10\% newborn calf serum and filtered through a collector tissue sieve (40 mesh; Fisher Scientific Co., Pittsburgh, PA). The islets were then purified by a density gradient (Histopaque-10077; Sigma Immunochemicals) centrifuged at 2,500 rpm for $20 \mathrm{~min}$. Islet yield was calculated by taking samples of $100 \mu \mathrm{l}$ from the final preparation. Aliquots of 2,000 islet equivalents each were then prepared. A single aliquot of 2,000 freshly isolated islet equivalents was aspirated into a $200-\mu l$ pipette tip (USA Scientific Plastics, Ocala, FL) connected with a 1-ml syringe (Hamilton Co., Reno, NE) and then transferred into polyethylene tubing P-50 (Becton Dickinson \& Co., Parsipanny, NJ). Under light methoxyflurane (Metofane; PitmanMoore Inc., Mundelein, IL) anesthesia, the left kidney of the recipient

1. Abbreviations used in this paper: $\mathrm{KpNPPase}, \mathrm{K}^{+}$-stimulated $p$-nitrophenol phosphatase; MsM, microsomal membrane; PM, plasma membrane; $R_{\mathrm{o}}$, plasma membrane glucose transporters measured by cytochalasin B binding; $V_{\max }$, glucose transport maximal velocity. rat was exposed through a lumbar incision. A capsulotomy was performed on the caudal outer surface of the kidney, and the tip of the tube was advanced under the capsule; the tubing was then removed and the capsulotomy was cauterized with a disposable low-temperature cautery pen (Surgicare, Dayville, CT).

After transplantation, the rats were bled every 2-5 d between 9:30 and 10:30 a.m. Blood samples were collected from the snipped tails using heparinized capillary tubes, and blood glucose was determined using a portable glucose meter (One Touch II; Johnson \& Johnson, Milpitas, CA).

Oral glucose load. $5 \mathrm{~d}$ before the terminal experiment (see below), six rats from the control group, six from the untreated diabetic group, and 10 from the transplanted diabetic group, after an overnight fast, received an oral glucose load ( $2 \mathrm{~g} / \mathrm{kg}$ body wt), half by spontaneous ingestion from the tip of a gavage needle and half by intragastric gavage. Heparinized blood samples were collected from the tail before and 30,60,90,120, and $180 \mathrm{~min}$ after the glucose ingestion and were used for plasma glucose determination (Yellow Springs Instruments, Yellow Springs, $\mathrm{OH}$ ).

Experimental day procedures. All animals were studied after an overnight fast between 8:30 and 10:30 a.m. Maximal insulin stimulation was achieved by intraperitoneal injection of $20 \mathrm{U}$ of insulin 30 min before killing the animals. The rats were considered insulin stimulated when serum insulin concentration exceeded $20,000 \mu \mathrm{U} / \mathrm{ml}$. Basal animals were handled but received no intraperitoneal injection. The animals were killed by a blow to the head followed by decapitation, and blood was collected for serum glucose and insulin determinations. Soleus, white and red gastrocnemius, and extensor digitorum longus muscles from both legs were quickly exposed and dissected, and fat and connective tissue was removed.

Plasma glucose was determined by glucose analyzer (Yellow Springs Instruments). Insulin concentration was determined with a double antibody radioimmunoassay using guinea pig anti-rat insulin serum, a rat insulin standard, and ${ }^{125}$ I-labeled insulin (Linco Research, St. Louis, MO).

Muscle membrane preparation. PM and MsM were prepared as previously described (4). Briefly, the dissected muscles, pooled together, were weighed and homogenized by mincing, polytroning, and homogenizing in a homogenizer (Potter-Elvejhem; A.H. Thomas Co., Swedesboro, NJ) using a Tris-sucrose buffer (255 mM sucrose, 100 $\mathrm{mM}$ Tris, $0.2 \mathrm{mM}$ EDTA, $\mathrm{pH}$ 7.6). After measuring the total volume, a small aliquot of the homogenate was taken and used later for $\mathrm{K}^{+}$stimulated $p$-nitrophenol phosphatase (KpNPPase), protein determination, and Western blotting. All of the following steps were performed at $4^{\circ} \mathrm{C}$ unless otherwise indicated. The homogenate was centrifuged at $34,000 \mathrm{~g}$ for $20 \mathrm{~min}$. The resulting pellet was used to prepare PM, while the supernatant was used for the preparation of MsM.

$\mathrm{PM}$ were prepared by resuspending the pellet in a sucrose/Hepes buffer $\left(250 \mathrm{mM}\right.$ sucrose, $20 \mathrm{mM}$ Hepes, $\mathrm{pH} 7.4$, at $\left.4^{\circ} \mathrm{C}\right)$. $\mathrm{KCl}$ and sodium pyrophosphate were added to final concentrations of $300 \mathrm{mM}$ and $25 \mathrm{mM}$, respectively. After centrifugation for $1 \mathrm{~h}$ at $227,000 \mathrm{~g}$, the resuspended pellet was incubated with DNAase for $1 \mathrm{~h}$ at $30^{\circ} \mathrm{C}$ and recentrifuged. The resulting pellet was resuspended in $34 \%$ sucrose with $20 \mathrm{mM}$ Hepes for a 16-h centrifugation in a sucrose step gradient. The plasma membranes were collected from the 12/27 and 27/ $30 \%$ sucrose interfaces. The membranes in these fractions were pelleted by centrifugation, resuspended in the sucrose/Hepes buffer to a final protein concentration of $2-4 \mathrm{mg} / \mathrm{ml}$, and stored in liquid $\mathrm{N}_{2}$ for later use.

MsM were prepared from the supernatant of the initial 34,000-g centrifugation. This supernatant was then recentrifuged at $227,000 \mathrm{~g}$ for $60 \mathrm{~min}$; the pellet was resuspended in a small volume of buffer containing $255 \mathrm{mM}$ sucrose, $1.0 \mathrm{mM}$ Tris- $\mathrm{HCl}$, and $1.0 \mathrm{mM} \mathrm{MgCl}_{2}, \mathrm{pH}$ 8.5 , at $4^{\circ} \mathrm{C}$, and layered on a discontinuous $0.9 \mathrm{M}$ sucrose gradient in $20 \mathrm{mM}$ Tris- $\mathrm{HCl}, 1 \mathrm{mM}$ EDTA, $\mathrm{pH} 7.4$, at $4^{\circ} \mathrm{C}$. The gradient tubes were centrifuged at $135,000 \mathrm{~g}$ for $60 \mathrm{~min}$, and the microsomal membrane band at the interface and all buffer above the band were di- 
luted in water and centrifuged at $227,000 \mathrm{~g}$ for $60 \mathrm{~min}$. The resulting pellet was resuspended in sucrose/Hepes buffer to a final concentration of $1-2 \mathrm{mg} / \mathrm{ml}$.

An aliquot from each suspension was removed and used for KpNPPase and protein determination, while the remaining sample was stored in liquid nitrogen until used for Western blotting, glucose transport activity, and cytochalasin B binding measurements (PM only).

Protein and marker enzyme assays. Homogenate, PM, and MsM protein content was determined for each preparation with the Coomassie brilliant blue method (Bio-Rad Laboratories, Richmond, CA) as described by Bradford (39) using BSA as standard. KpNPPase specific activity was assayed in the absence or presence of $20 \mathrm{mM} \mathrm{K}^{+}$as a PM marker $(40,41)$. KpNPPase recovery in PM or MsM fractions was calculated by dividing the total activity collected in the PM or the MsM fraction by the total activity in the homogenate. The total activity was obtained by multiplying the KpNPPase activity per milligram of protein by the total amount of proteins in the homogenate and in the PM and MsM fractions. KpNPPase enrichment was calculated by dividing the KpNPPase activity per milligram of protein in the PM, or in the MsM, by that in the homogenate.

Glucose transport. The uptake of ${ }_{\mathrm{D}-}\left[{ }^{14} \mathrm{C}(\mathrm{U})\right]$-glucose and $\mathrm{L}-\left[{ }^{3} \mathrm{H}\right.$ $(\mathrm{N})$ ]-glucose uptake in plasma membrane vesicles was determined under equilibrium exchange conditions at $25^{\circ} \mathrm{C}$ using a rapid filtration technique, as previously described $(3,21)$. PM were preequilibrated with a Hepes-buffered Krebs-Ringer solution containing 1, 5, 10, 20, 40 , or $60 \mathrm{mM} \mathrm{L-}$ and D-glucose. Uptake was measured at four time points for each preparation ( $0-5 \mathrm{~s})$. Transport was initiated by combining an aliquot of the membranes with Hepes-buffered Krebs solution and 1-60 mM L- and D-glucose with $6 \mu \mathrm{Ci}$ of L- $\left[{ }^{3} \mathrm{H}(\mathrm{N})\right]$ glucose and $1.6 \mu \mathrm{Ci}$ of $\mathrm{D}^{-}\left[{ }^{14} \mathrm{C}(\mathrm{U})\right]$ glucose (New England Nuclear-DuPont, Boston, MA). Transport was stopped by the addition of Hepes-buffered Krebs solution containing $0.2 \mathrm{mM}$ phloretin at $4^{\circ} \mathrm{C}$. The membranes were rapidly loaded onto a filter (HA $0.45 \mathrm{~mm}$; Millipore Corp., Bedford, MA) and washed, and the filter and adhering membranes were analyzed by liquid scintillation counting using quench correction for the dual label. Facilitated transport was calculated by subtracting the initial rate of L-glucose influx from that of D-glucose. $V_{\max }$ and $K_{\mathrm{m}}$ were determined using nonlinear least-squares fit of the data (42).

Cytochalasin B binding. Equilibrium D-glucose-inhibitable $\left[{ }^{3} \mathrm{H}\right] \mathrm{cy}-$ tochalasin $\mathrm{B}$ binding was measured and the concentration of glucose transporters was determined by a modification of the method of Wardzala et al. (43) as previously described (4). Scatchard plots were generated from binding studies in which membranes were incubated with cytochalasin B in the presence or absence of $500 \mathrm{mM}$ D-glucose. Cytochalasin $\mathrm{E}(2 \mu \mathrm{M})$ was present to decrease nonspecific binding. Bound cytochalasin B was separated from free cytochalasin B by centrifugation. Tracer amounts of $\left[{ }^{14} \mathrm{C}\right]$ urea were used to correct for $\left[{ }^{3} \mathrm{H}\right]$ cytochalasin B trapped in the pellet. The total number of glucose transporters $\left(R_{\mathrm{o}}\right)$ and the dissociation constant $\left(K_{\mathrm{d}}\right)$ were determined from a linear plot derived by subtraction along the radial axes of binding curves generated in the presence of D-glucose from those in the absence of D-glucose.

Carrier turnover number. The carrier turnover number $(3,21$, 28 ), an index of glucose transported per transporter, was calculated by dividing the glucose transport $V_{\max }$ by the total number of glucose transporters, both measured in the same plasma membrane fraction $\left(V_{\max } / R_{\mathrm{o}}\right)$. Carrier turnover number represents the average activity of transporters in the PM. Therefore, changes in transporter isoforms in the PM, if they had different intrinsic activity, would result in an altered average carrier turnover number even if no change of individual transporter intrinsic activity occurred.

Western blotting. Homogenate (200 $\mu \mathrm{g}$ protein for GLUT4), PM, and MsM (30 $\mu \mathrm{g}$ for GLUT4, $65 \mu \mathrm{g}$ for GLUT1) were subjected to SDS-PAGE, and resolved proteins were transferred $(44,45)$ to polyvinylidene fluoride filters (Corning Costar Corp., Cambridge, MA). Incubations were carried out in Tris-buffered saline (20 mM Tris, 250
$\mathrm{mM} \mathrm{NaCl}, \mathrm{pH} 7.5)$ at $22^{\circ} \mathrm{C}$ unless otherwise indicated. The polyvinylidene fluoride transfer membranes were blocked in Tris-buffered saline containing $0.5 \%$ Tween $20,3 \%$ BSA, $0.01 \%$ Na azide. For GLUT1 determination, nonfat dry milk ( $2 \%$ final concentration; Carnation) was added to this blocking buffer. To identify GLUT4 or GLUT1 proteins, the transfer membranes were incubated with either a polyclonal antibody (GT4 at a concentration of $5 \mu \mathrm{g} / \mathrm{ml}$, produced from a synthetic peptide corresponding to a 15 -amino acid $\mathrm{COOH}$ terminal sequence in rat muscle GLUT4, gift of Dr. Robert J. Smith, Joslin Diabetes Center, Harvard Medical School, Boston, MA) or the polyclonal antibody (East Acres Biologicals, Southbridge, MA [46], produced from a synthetic peptide corresponding to a 12-amino acid $\mathrm{COOH}$-terminal sequence in rat brain GLUT1) at the dilution 1:200, followed by incubation with ${ }^{125}$ I-labeled protein A (ICN, Irvine, CA). The polyvinylidene fluoride filters were analyzed, and bands corresponding to specific glucose transporters were quantitated by a PhosphorImager (Molecular Dynamics, Inc., Sunnyvale, CA). For each gel, serial dilutions of a microsomal membrane stock were run as an internal standard to allow a comparison of samples run in separate gels and to determine linearity.

Statistical analysis. All data were compared using SAS (Statistical Analysis System, Cary, NC) programs. Group means were compared by two-way ANOVA using the General Linear Models Procedure with least-squares means for the among-groups comparisons. The differences between groups were accepted to be significant when $P<$ 0.05 . Data are expressed as mean $\pm \mathrm{SE}$.

To simplify the graphic representation of the data, the statistical probabilities shown in the figures are all indicated with the highest significant $P$ value in that particular figure, although the majority of the $P$ values are much lower.

\section{Results}

General characteristics of the rats studied. The nonfasted blood glucose levels measured in the diabetic animals after the transplantation are shown in Fig. 1. As shown in this graph, blood glucose decreased in all the animals, although the pattern of this decrease was different among them. Fig. 2 shows plasma glucose concentrations in response to the oral glucose load. In the basal fasting state, plasma glucose was significantly higher in the untreated diabetic animals and remained elevated for the $3 \mathrm{~h}$ after glucose ingestion. Basal fasting plasma glucose concentrations were not elevated in the transplanted diabetic animals; however, the glucose load induced a significantly greater increase in the plasma glucose concentration compared with controls. Therefore, the transplanted diabetic rats were characterized by an impaired response to the glucose load, even though their basal glucose concentrations were normal.

On the day of the experiment, serum glucose concentrations were much higher in the untreated diabetic rats in the basal fasting state $(110 \pm 5,452 \pm 9$, and $102 \pm 3 \mathrm{mg} / \mathrm{dl}$ in controls, diabetics, and transplanted diabetics, respectively). In the basal fasting state, serum insulin levels were not significantly different in the three groups $(38 \pm 12,18 \pm 6$, and $28 \pm 3$ $\mu \mathrm{U} / \mathrm{ml}$ in controls, diabetics, and transplanted diabetics, respectively; $P=0.16$ among the groups). Compared with controls, body weight was much lower in the diabetic rats (348 $\pm 10,246 \pm 9$, and $302 \pm 5 \mathrm{~g}$ in controls, diabetics, and transplanted diabetics, respectively).

Enzymatic characterization of PM and MsM. Table I shows KpNPPase activity in PM, MsM, and starting homogenate. There were no differences in KpNNpase activity, recovery, and enrichment in the PM fraction from the groups. A very low level of KpNPPase activity was detected in the in MsM 


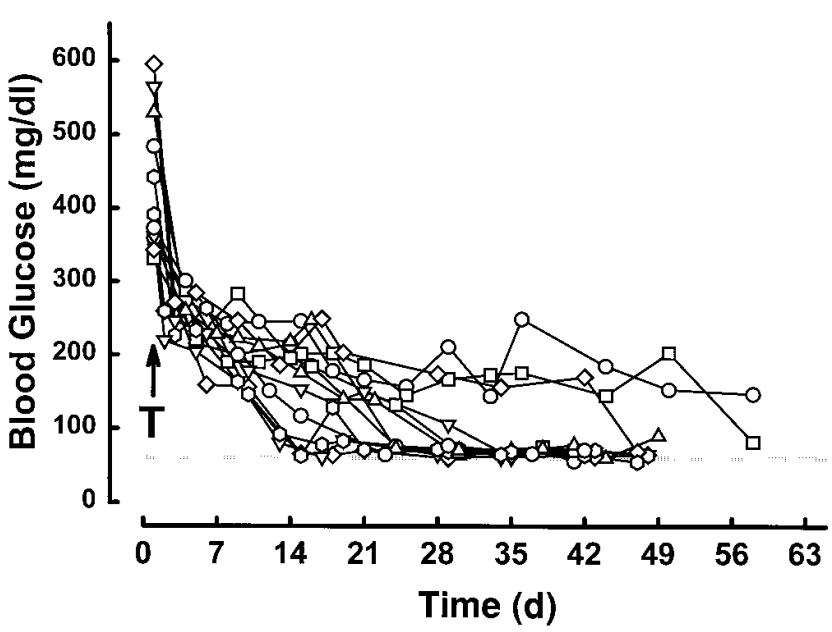

Figure 1. Blood glucose in the diabetic animals after islet transplantation. Blood samples were collected from the snipped tails and blood glucose was determined using a portable glucose meter. The shaded area represents the mean $\pm \mathrm{SE}$ of the measurements made in the 10 control rats.

fraction, indicating a low degree of contamination with PM in this fraction. No differences in KpNPPase activity, recovery, and enrichment were observed in the MsM fraction from the different groups. These data indicate that there were no differences in quality and purity of these fractions among the groups.

Glucose transport in the PM. Fig. $3 A$ shows basal and insulin-stimulated glucose transport $V_{\max }$, obtained in the plasma membrane vesicles from control, untreated diabetic, and transplanted diabetic animals. In the basal fasting state, glucose transport $V_{\max }$ was reduced $50 \%$ in the untreated diabetic animals compared with the control rats $(P<0.005)$. Islet transplantation restored the basal glucose transport $V_{\max }$ in the diabetic rats $(P=0.2$ vs controls and $P<0.03$ vs untreated diabetic rats). Insulin stimulation significantly increased the glucose transport $V_{\max }$ in all the groups studied $(P<0.005$ for all vs corresponding basal values). However, compared with the controls, glucose transport $V_{\max }$ was significantly reduced in the untreated diabetic animals $(18.7 \pm 1.5$ and $11.8 \pm 3.29$

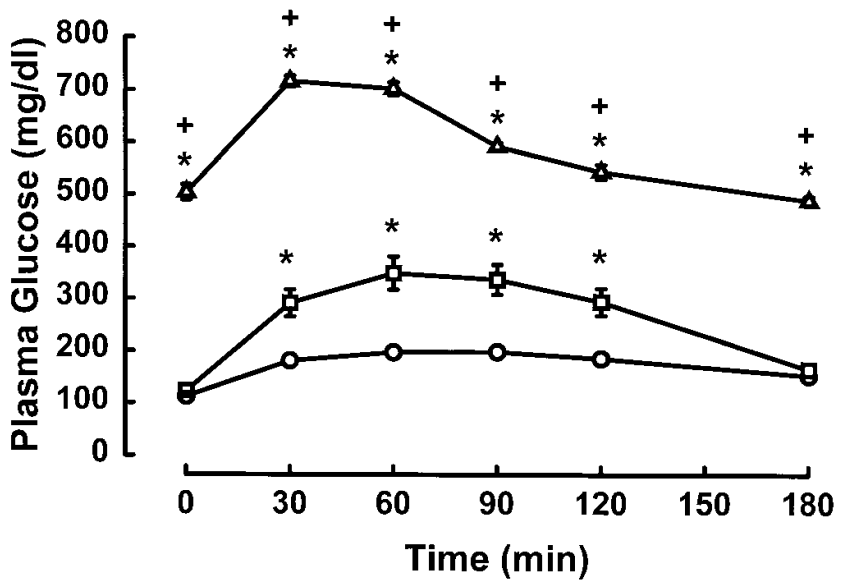

Figure 2. Plasma glucose before and after an oral glucose load $(2 \mathrm{~g} / \mathrm{kg}$ body wt) in control (circles), untreated diabetic (triangles), and transplanted diabetic (squares) animals. Plasma glucose was determined by glucose analyzer. Data are expressed as mean \pm SE. $* P<0.005$ vs controls; ${ }^{+} P<0.005$ vs transplanted diabetics.

$\mathrm{nmol} \cdot \mathrm{mg}^{-1} \cdot \mathrm{s}^{-1}$, respectively; $\left.P=0.01\right)$. Islet transplantation in the diabetic rats restored the normal insulin stimulated $V_{\max }$ $\left(19.3 \pm 2.25 \mathrm{nmol} \cdot \mathrm{mg}^{-1} \cdot \mathrm{s}^{-1} ; P=0.8 \mathrm{vs}\right.$ controls and $P<0.005$ vs untreated diabetic animals). In the basal fasting state, the $K_{\mathrm{m}}$ of the glucose transport was $62 \pm 6,30 \pm 4$, and $42 \pm 6 \mathrm{mM}$, in control, untreated diabetic, and transplanted diabetic rats. After insulin stimulation no change in the $K_{\mathrm{m}}$ was observed in any group, and no differences were present among the three groups $(45.9 \pm 11,24.2 \pm 5.5$, and $41.3 \pm 6 \mathrm{mM}$, in control, untreated diabetic, and transplanted diabetic rats, respectively).

Total glucose transporter number in the $P M\left(R_{o}\right)$. In the basal fasting state, diabetes decreased the total number of glucose transporters in the PM by $40 \%(P<0.05)$ (Fig. $3 B$ ). Islet transplantation completely restored the total number of glucose transporters in the PM $(P=0.8$ vs controls; $P<0.03$ vs untreated diabetic rats). Insulin stimulation, which induced a significant increase in the $R_{\mathrm{o}}$ in the control rats (from 10.2 \pm 0.6 to $17.8 \pm 2.0 \mathrm{pmol} \cdot \mathrm{mg}^{-1} ; P<0.01 \mathrm{vs}$ basal), did not induce a significant transporter translocation in the untreated diabetic animals $\left(9.6 \pm 1.7 \mathrm{pmol} \cdot \mathrm{mg}^{-1} ; P=0.2\right.$ vs basal; $P=0.006$ vs

Table I. KpNPPase Activity in Skeletal Muscle PM and MsM from the Rats Studied

\begin{tabular}{|c|c|c|c|c|c|c|}
\hline \multirow[b]{2}{*}{ Groups } & \multicolumn{2}{|c|}{ Controls } & \multicolumn{2}{|c|}{ Diabetics } & \multicolumn{2}{|c|}{ Transplanted } \\
\hline & Basal & Insulin & Basal & Insulin & Basal & Insulin \\
\hline \multicolumn{7}{|l|}{ Homogenate } \\
\hline Specific activity $\left(\mathrm{nmol} \cdot \mathrm{mg}^{-1} \cdot 30 \mathrm{~min}^{-1}\right)$ & $174 \pm 6.7$ & $190 \pm 12$ & $159 \pm 11$ & $124 \pm 9.5$ & $158 \pm 17$ & $141 \pm 6.1$ \\
\hline \multicolumn{7}{|l|}{ Plasma membranes } \\
\hline Specific activity $\left(\mathrm{nmol} \cdot \mathrm{mg}^{-1} \cdot 30 \mathrm{~min}^{-1}\right)$ & $3,186 \pm 199$ & $3,671 \pm 293$ & $3,147 \pm 317$ & $2,697 \pm 274$ & $3,062 \pm 194$ & $2,589 \pm 302$ \\
\hline Recovery (\%) & $6.5 \pm 0.6$ & $5.9 \pm 0.8$ & $6.4 \pm 1.2$ & $6.2 \pm 0.8$ & $5.6 \pm 0.7$ & $4.1 \pm 0.7$ \\
\hline Enrichment (fold) & $18.4 \pm 1.3$ & $19.5 \pm 1.5$ & $20.6 \pm 3.0$ & $19.9 \pm 2.0$ & $21.0 \pm 3.1$ & $15.7 \pm 1.6$ \\
\hline \multicolumn{7}{|l|}{ Microsomal membranes } \\
\hline Specific activity $\left(\mathrm{nmol} \cdot \mathrm{mg}^{-1} \cdot 30 \mathrm{~min}^{-1}\right)$ & $333 \pm 67$ & $398 \pm 65$ & $380 \pm 39$ & $385 \pm 31$ & $415 \pm 44$ & $423 \pm 42$ \\
\hline Recovery (\%) & $0.3 \pm 0.07$ & $0.3 \pm 0.08$ & $0.4 \pm 0.08$ & $0.5 \pm 0.02$ & $0.4 \pm 0.05$ & $0.4 \pm 0.08$ \\
\hline Enrichment (fold) & $1.95 \pm 0.4$ & $2.16 \pm 0.4$ & $2.39 \pm 0.3$ & $2.83 \pm 0.1$ & $2.78 \pm 0.4$ & $2.76 \pm 0.4$ \\
\hline
\end{tabular}



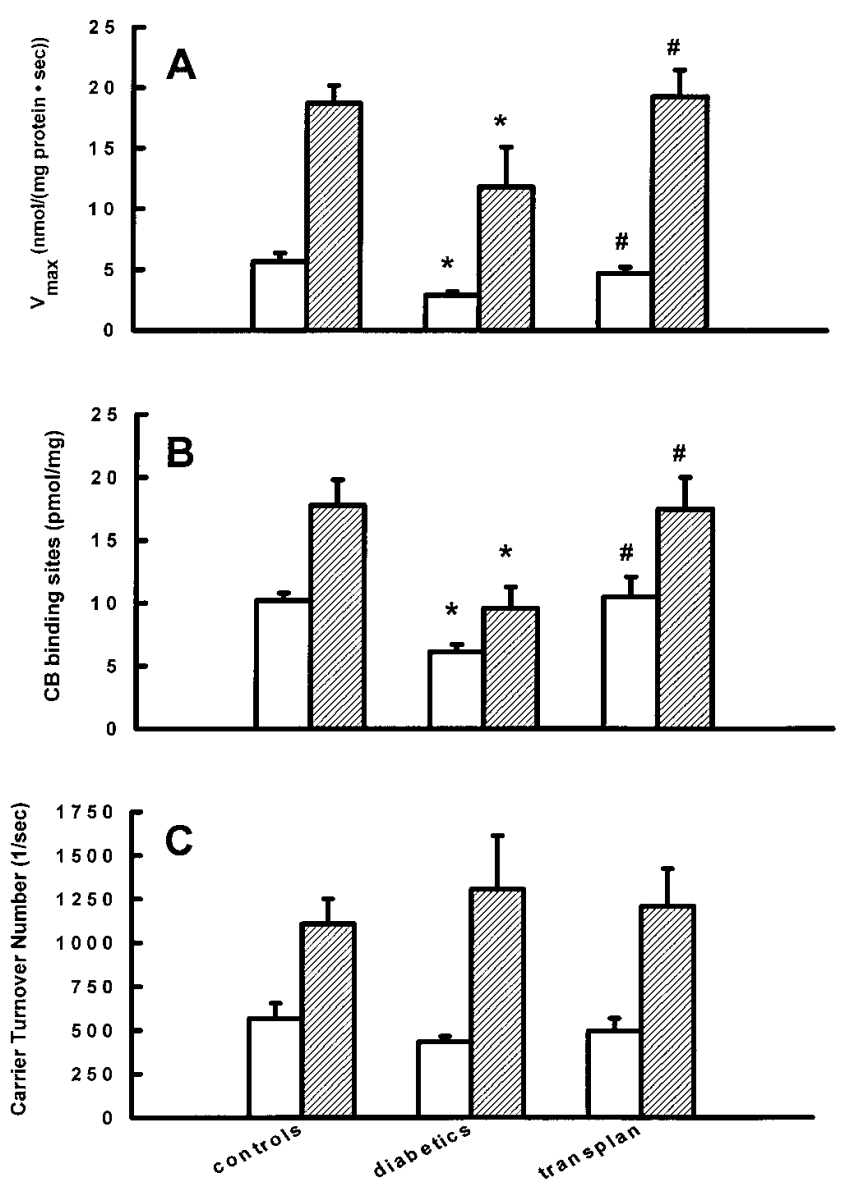

Figure 3. Glucose transport $V_{\max }(A)$, total glucose transporter number $\left(R_{\mathrm{o}}\right)(B)$, and carrier turnover number $(C)$ in skeletal muscle PM vesicles from rats studied in the basal fasting state (open bars) or after maximal insulin stimulation (striped bars). $* P<0.05$ vs control group; ${ }^{\sharp} P<0.05$ vs untreated diabetics.

controls). The $R_{\mathrm{o}}$ in the PM increased normally after insulin stimulation in the transplanted diabetic animals $(17.5 \pm 2.5$ pmol $\cdot \mathrm{mg}^{-1} ; P<0.005$ vs basal, $P=0.9$ vs controls, $P=0.006$ vs untreated diabetics).

Carrier turnover number. The carrier turnover number, obtained by dividing the $V_{\max }$ by the $R_{\mathrm{o}}$, is an index of the average activity of glucose transporters in the PM fraction. In Fig. 3 $C$, the carrier turnover number data are shown. Although a slight decrease could be observed in the untreated diabetic animals, there were no statistically significant differences among the three groups $\left(568 \pm 85,434 \pm 31\right.$, and $495 \pm 74 \mathrm{~s}^{-1}$, in control, untreated diabetic, and transplanted diabetic rats, respectively). Insulin stimulation induced a significant increase in the carrier turnover number in all the groups.

GLUT4 and GLUT1 transporters. In addition to measuring the PM $R_{\mathrm{o}}$, we also determined the effects of diabetes and transplantation on the distribution of the two glucose transporter isoforms expressed in skeletal muscle, GLUT4 and GLUT1. In the basal fasting state PM GLUT4 was reduced in the untreated diabetic rats $(P<0.005)$ (Fig. 4). Islet transplantation restored the PM GLUT4 content in the diabetic animals $(P=0.3$ vs controls, and $P<0.03$ vs untreated diabetics). Insulin stimulation increased PM GLUT4 content $50 \%$ above the basal level in the control group $(P<0.05)$, while no significant translocation of GLUT4 could be detected in the untreated diabetic rats $(0.5 \pm 0.1$ arbitrary units; $P=0.4$ vs basal). Islet transplantation restored normal GLUT4 translocation in the diabetic animals $(1.25 \pm 0.1$ arbitrary units; $P<0.03$ vs basal). Compared with the controls, the insulin-stimulated PM GLUT4 content was significantly reduced in the untreated diabetic animals $(P<0.001)$ and normal in the transplanted diabetic rats $(P=0.3$ vs controls; $P<$ 0.03 vs untreated diabetics). In the intracellular microsomal membrane fraction, basal fasting GLUT4 content was decreased in the untreated diabetic animals (Fig. 4). Microsomal membrane GLUT4 was normalized by transplantation $(P=$ 0.3 vs controls, $P<0.005$ vs untreated diabetics). In the insulin-stimulated state, MsM content of GLUT 4 was significantly reduced in the untreated diabetics and normal in the transplanted diabetic animals. However, it is particularly interesting that the reduction in GLUT4 content in the MsM after insulin stimulation appeared to be similar in the three groups, although the recruitment of GLUT4 to the PM was impaired in the untreated diabetic rats.

Compared with controls, the homogenate GLUT4 content was reduced in the untreated diabetic rats $(1.0 \pm 0.06$ and $0.5 \pm 0.04$ arbitrary units, respectively; $P<0.001)$ and normal in the transplanted diabetic animals $(0.91 \pm 0.04$ arbitrary units; $P=$ NS vs controls, $P<0.001$ vs untreated diabetics $)$.

No differences in PM GLUT1 content were detected among the groups, either in the basal fasting state or after insulin stimulation (Fig. 5). However, the MsM content of GLUT1 was increased in the untreated diabetics compared with the controls $(1.4 \pm 0.1$ and $0.85 \pm 0.3$ arbitrary units, respectively; $P<$ $0.01)$, and normal in the transplanted diabetic rats $(P=0.76$ vs

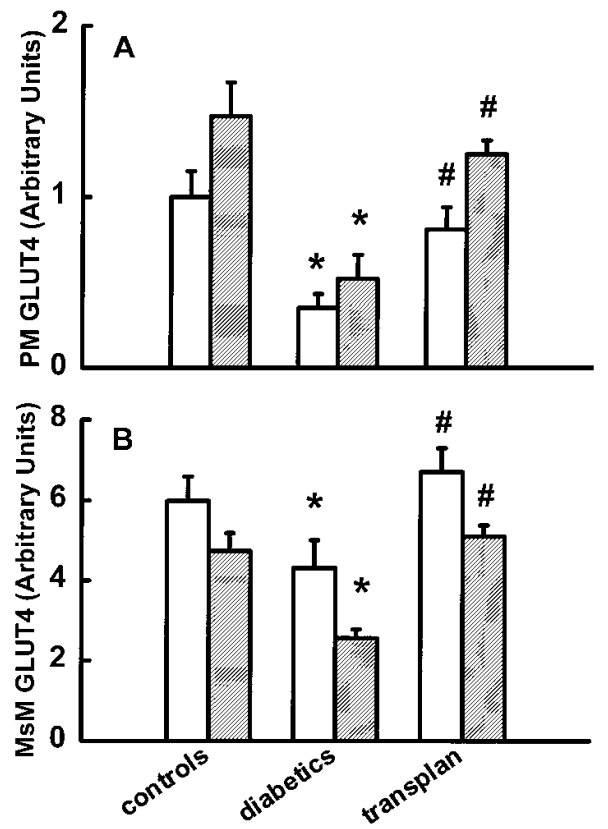

Figure 4. PhosphorImager analysis of Western blots of skeletal muscle PM $(A)$ and MsM $(B)$ GLUT4 protein from control and diabetic animals in the basal fasting state (open bars) or after maximal insulin stimulation (striped bars). The data are expressed as arbitrary units of a reference standard used for the comparisons among different blots. Results are mean \pm SE. ${ }^{*} P<0.05$ vs the control group; ${ }^{\#} P<0.05$ vs the untreated diabetic group. 


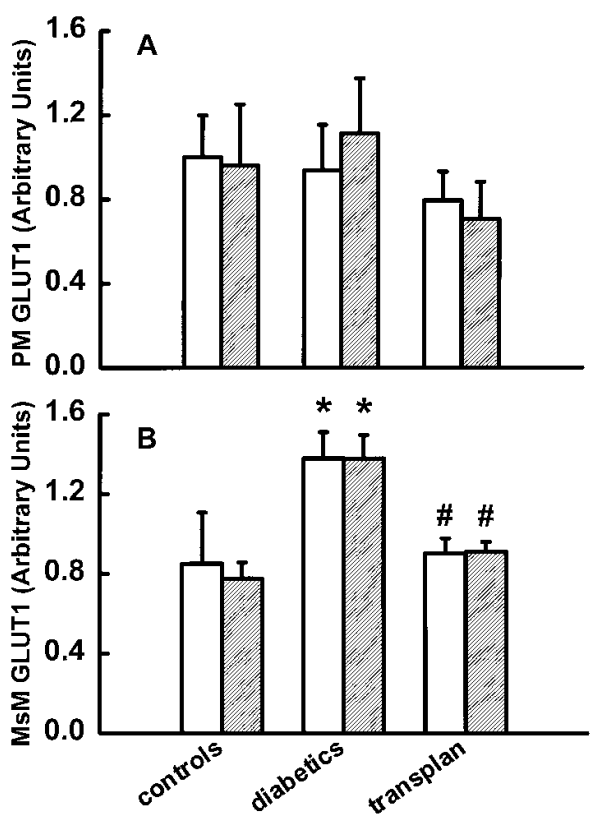

Figure 5. PhosphorImager analysis of Western blots of skeletal muscle PM $(A)$ and MsM $(B)$ GLUT1 protein from control and diabetic animals in the basal fasting state (open bars) or after maximal insulin stimulation (striped bars). The data are expressed as arbitrary units of a reference standard used for the comparisons among different blots. Results are mean \pm SE. $* P<0.01$ vs the control group; ${ }^{*} P<0.01$ vs the untreated diabetic group.

controls; $P<0.01$ vs untreated diabetics). No effect of insulin on PM or MsM GLUT1 content was observed.

\section{Discussion}

We show that islet transplantation fully corrected impaired skeletal muscle glucose transport $V_{\max }$ in streptozocin diabetes, demonstrating that adequate insulin delivery, although into the peripheral circulation, is capable of normalizing the glucose flux through the PM. In addition, islet transplantation restored insulin-induced transporter translocation to normal in skeletal muscle. Since no differences in the insulin-induced decrease in MsM GLUT4 content were observed among the three groups studied, the normalization of GLUT4 translocation in diabetic animals after islet transplantation presumably was at least partially due to the improved recruitment of transporters to the PM. Furthermore, we found a specific increase in MsM content of GLUT1 with diabetes, and we show that this increase was corrected by islet transplantation.

The inability of insulin therapy to normalize insulin-stimulated glucose transport and transporter translocation previously reported (33) could be due to several reasons. First, the insulin dose was not sufficient to reduce plasma glucose concentration to normal. Because chronic hyperglycemia affects insulin-stimulated skeletal muscle glucose transport (28), and transporter translocation (28), the inability of insulin therapy to restore these parameters to normal might reside in the residual hyperglycemia. Second, insulin therapy by subcutaneous injections in rats could be associated with hypoglycemic episodes that, through the activation of a counterregulatory response, might induce an impairment of insulin-mediated skeletal muscle glucose uptake $(47,48)$. Third, to obtain the normalization of plasma glucose by subcutaneous insulin administration, it is necessary to increase peripheral insulin concentration to a level higher than normal, because of the absence of the physiological ratio between portal vein and peripheral circulation levels. These increased levels have been postulated to cause resistance to the acute effect of insulin on the skeletal muscle glucose uptake (34-36). In the current study, fasting insulin concentrations in streptozocin diabetic rats treated with islet transplantation were comparable to that of the control rats. However, if plasma insulin levels had been measured in the fed state, peripheral hyperinsulinemia might have been found. Even though peripheral hyperinsulinemia has been postulated to cause insulin resistance (32-36) and has been associated with enhanced insulin action in adipocytes (23), no evidence for either effect was found in this study.

Theoretically, the liver may participate in the regulation of muscle glucose transport. It could be that an adequate liver insulinization is necessary for release and/or uptake of substrates involved in the regulation of skeletal muscle glucose uptake. We did not measure insulin levels in the portal vein of the diabetic rats, but we assume they were lower than in the control rats. Therefore, our results demonstrate that the normal ratio between portal and peripheral levels of insulin is not necessary for the normalization of skeletal muscle glucose transport in rats. Since glucose transport is the rate-limiting step for glucose uptake under physiological circumstances (49-53), our data suggest that islet transplantation might fully correct the defect in insulin-stimulated glucose disposal. The impaired glucose tolerance detected in this study in the transplanted diabetic rats (Fig. 2) may be due to inadequate suppression of hepatic glucose output, perhaps because of lower portal insulin concentrations. Also, we cannot assume that glucose-induced insulin secretion was completely normal in these transplanted rats. The few data available on diabetic patients after pancreas transplantation show that transplantation did not normalize insulin-mediated whole body glucose uptake (54) or muscle GLUT4 content (55). However, the data from patients after pancreas transplantation are difficult to interpret, because of immunosuppressive therapy that could be responsible for decreased muscle glucose uptake (54).

As shown in several different cell types (56-61), the number of glucose transporters in the PM is regulated by the relative rates of exocytosis and endocytosis of glucose transporters from/to one or more interconnected intracellular compartments. Insulin stimulation increases GLUT4 in the PM mainly by increasing the rate of exocytosis and only minimally reducing the rate of endocytosis (60), thus resulting in the net translocation of GLUT4 from an intracellular pool to the PM. Interestingly, data obtained with the subcellular fractionation procedure (5), confirmed in the current study, show that, although the whole muscle content of GLUT4 is reduced, the impaired insulin-induced GLUT4 translocation to PM in the muscle of streptozocin diabetic rats is associated with a concomitant normal decrease of the GLUT4 content in the intracellular microsomal pool. Therefore, it is possible that GLUT4 transporters leave the intracellular pool normally but migrate to a compartment other than the PM, not selected by the fractionation procedures (62). Alternatively, the lack of an insulinstimulated increase of glucose transporters on the PM in diabetes could be due to a reduced ability of insulin to inhibit endocytosis, as was recently suggested for adipocytes (63). 
Thus, it is possible that the acute insulin signal "reaches" the MsM pool in streptozocin diabetic rats, as suggested by the fact that insulin-stimulated IRS-1 phosphorylation and total PI-3 kinase activity are increased rather than decreased (64, $65)$. The presence of hyperglycemia might directly interfere with the targeting of GLUT4 to the PM and/or increase its endocytosis, as suggested by the improvement of GLUT4 translocation induced by phlorizin without changing muscle GLUT4 content (28). In the current study, we show that islet transplantation normalizes the whole muscle content of GLUT4 and corrects the defect in translocation of this transporter to the PM. Therefore, only the association of normal glucose and insulin levels may completely restore muscle GLUT4 content, the translocation process, and therefore glucose transport and uptake.

Basal state GLUT1 content in the PM was not changed by diabetes, in agreement with previous observations by our group (28) and others (5). In addition, we show for the first time a marked increase in MsM content of GLUT1 in diabetic rats (Fig. 5). In contrast with these data, Dimitrakoudis et al. (66) found an increase in GLUT1 content in PM in streptozocin diabetes. However, Dimitrakoudis et al. (66), using the fractionation procedure of Klip et al. (67), detected the largest increase of GLUT1 in a fraction in which no increase in the number of glucose transporters with insulin stimulation was observed in control rats (62). Perhaps this fraction is very rich in microsomal membranes containing glucose transporters. Thus, our observation that GLUT1 increases in diabetes specifically in the MsM fraction may not be in conflict with those data $(62,66)$.

Insulin-stimulated skeletal muscle glucose uptake is impaired in human diabetes (8-12). However, the few data available show that this impairment is not associated with a reduction in PM GLUT4 content (19). Therefore, a major role could be played by reduction in transporter intrinsic activity. We have previously demonstrated that the decrease in transporter intrinsic activity has a relevant role in the impairment of basal skeletal muscle glucose transport in nonfasted streptozocin diabetic rats (28). A marked reduction of hyperglycemia in these rats, obtained by phlorizin administration, did not interfere with the decrease of carrier turnover number (28), suggesting that hyperglycemia did not play a relevant role in the impairment of transporter intrinsic activity. We reanalyzed the data from that study (28) by correlating serum insulin concentration and carrier turnover number for both the diabetic and the control animals studied in the basal nonfasted state. This analysis showed that serum insulin concentration and carrier turnover number positively and significantly correlated $(n=36, r=0.4$, $P=0.01$ ) (excluded from this analysis were diabetic rats that received the streptozocin injection $24 \mathrm{~h}$ before the experiment, because of the severe fluctuation in insulin levels in the few hours immediately preceding the experiment [68, 69]), suggesting that basal serum insulin concentration modulates transporter intrinsic activity. In the current study, to test further the hypothesis that hypoinsulinemia was responsible for the impairment of basal carrier turnover number in diabetic rats (28), we examined the animals in the basal fasting state, when insulin levels were not different among the groups. Compared with the controls, we found no differences in the carrier turnover number in the untreated diabetic rats. Furthermore, when large amounts of insulin were administered to both diabetic and control animals, no differences in carrier turnover number were present, either in the current or in our previous study (28). Taken together, these data support the hypothesis that the decrease in carrier turnover number in streptozocin diabetic rats is due to the insulin deficiency and that this impairment is quickly reversed by changes in serum insulin concentration.

In conclusion, our data show that islet transplantation under the kidney capsule completely restores the impaired skeletal muscle glucose transport system of streptozocin diabetic rats, supporting the hypothesis that the route of insulin delivery is not a significant variable. Our data suggest also that the impairment in basal transporter turnover number in streptozocin diabetes is due to the insulin deficiency.

\section{Acknowledgments}

We thank Dr. R.J. Smith for the gift of the GLUT4 antibody, Dr. C.J. de Sousa for performing the insulin assay, and J. Hollister for isolation of islets.

This work was supported by U.S. Public Health Service grant RO1-DK-26317 (to E.S. Horton) and a grant from the Joslin Diabetes Center. Other funding was from U.S. Public Health Service grants DK-35449 (to G.C. Weir) and DK 36836 (Joslin Diabetes Endocrinology Research Centers), and from the Jesse B. Cox Foundation (to G.C. Weir). R. Napoli was on leave from "Federico II" University Medical School, Napoli, Italy, and supported by the American Diabetes Association Mentor-based Postdoctoral Fellowship Program (to E.S. Horton) and a Mary K. Iacocca Fellowship from the Joslin Research Laboratory. A.M. Davalli was on leave from Istituto Scientifico San Raffaele, Milano, Italy, and supported by a Juvenile Diabetes Foundation International Postdoctoral Fellowship.

\section{References}

1. Kahn, B.B., L. Rossetti, H.F. Lodish, and M.J. Charron. 1991. Decreased in vivo glucose uptake but normal expression of GLUT1 and GLUT4 in skeletal muscle of diabetic rats. J. Clin. Invest. 87:2197-2206.

2. Calderhead, D.M., K. Kitagawa, G.E. Lienhard, and G.W. Gould. 1990. Translocation of the brain-type glucose transporter largely accounts for insulin stimulation of glucose transport in BC3H-1 myocytes. Biochem. J. 269:597-601.

3. King, P.A., E.D. Horton, M.F. Hirshman, and E.S. Horton. 1992. Insulin resistance in obese Zucker rat (fa/fa) skeletal muscle is associated with a failure of glucose transporter translocation. J. Clin. Invest. 90:1568-1575.

4. Hirshman, M.F., L.J. Goodyear, L.J. Wardzala, E.D. Horton, and E.S. Horton. 1990. Identification of an intracellular pool of glucose transporters from basal and insulin-stimulated rat skeletal muscle. J. Biol. Chem. 265:987991.

5. Klip, A., T. Ramlal, P.J. Bilan, G.D. Cartee, E.A. Gulve, and J.O. Holloszy. 1990. Recruitment of GLUT-4 glucose transporters by insulin in diabetic rat skeletal muscle. Biochem. Biophys. Res. Commun. 172:728-736.

6. Goodyear, L.J., M.F. Hirshman, P.M. Valyou, and E.S. Horton. 1992. Glucose transporter number, function, and subcellular distribution in rat skeletal muscle after exercise training. Diabetes. 41:1091-1099.

7. Douen, A.G., T. Ramlal, S. Rastogi, P.J. Bilan, G.D. Cartee, M. Vranic, J.O. Holloszy, and A. Klip. 1990. Exercise induces recruitment of the "insulinresponsive glucose transporter." Evidence for distinct intracellular insulin- and exercise-recruitable transporter pools in skeletal muscle. J. Biol. Chem. 265: 13427-13430.

8. Capaldo, B., R. Napoli, L. Di Marino, A. Picardi, G. Riccardi, and L. Sacca'. 1988. Quantification of forearm glucose and free fatty acid (FFA) disposal in normal subjects and type II diabetic patients: evidence against an essential role for FFA in the pathogenesis of insulin resistance. J. Clin. Endocrinol. \& Metab. 67:893-898.

9. DeFronzo, R.A., R. Hendler, and D. Simonson. 1982. Insulin resistance is a prominent feature of insulin-dependent diabetes. Diabetes. 31:795-801.

10. Yki-Jarvinen, H., and V.A. Koivisto. 1984. Insulin sensitivity in newly diagnosed type I diabetics after ketoacidosis and after three months of insulin therapy. J. Clin. Endocrinol. \& Metab. 287:659-666.

11. DeFronzo, R.A., R. Gunnarson, O. Bjorkman, M. Olsson, and J. Warren. 1985. Effects of insulin on peripheral and splanchnic glucose metabolism in non-insulin dependent (type II) diabetes mellitus. J. Clin. Invest. 76:149-155.

12. Baron, A.D., M. Laakso, G. Brechtel, and S.V. Edelman. 1991. Reduced 
capacity and affinity of skeletal muscle for insulin-mediated glucose uptake in noninsulin dependent diabetic subjects. Effects of insulin therapy. J. Clin. Invest. 87:1186-1194.

13. Bonadonna, R., S. Del Prato, M.P. Saccomanni, E. Bonora, G. Gulli, E. Ferrannini, D. Bier, C. Cobelli, and R.A. DeFronzo. 1993. Transmembrane glucose transport in skeletal muscle of patients with non-insulin-dependent diabetes. J. Clin. Invest. 92:486-494.

14. Pedersen, O., J.F. Bak, P.H. Andersen, S. Lund, D.E. Moller, J.S. Flier, and B.B. Kahn. 1990. Evidence against altered expression of GLUT1 or GLUT4 in skeletal muscle of patients with obesity or NIDDM. Diabetes. 39: $865-870$.

15. Handberg, A., A. Vaag, P. Damsbo, H. Beck Nielsen, and J. Vinten. 1990. Expression of insulin regulatable glucose transporters in skeletal muscle from type 2 (non-insulin-dependent) diabetic patients. Diabetologia. 33:625627.

16. Kahn, B.B., A.S. Rosen, J.F. Bak, P.H. Andersen, S. Lund, and O. Pedersen. 1992. Expression of GLUT1 and GLUT4 glucose transporters in skeletal muscle of humans with insulin-dependent diabetes mellitus: regulatory effects of metabolic factors. J. Clin. Endocrinol. \& Metab. 74:1101-1109.

17. Andersen, P.H., S. Lund, H. Vestergaard, S. Junker, B.B. Kahn, and O. Pedersen. 1993. Expression of the major insulin regulatable glucose transporter (GLUT4) in skeletal muscle on non insulin dependent diabetic patients and healthy subjects before and after insulin infusion. J. Clin. Endocrinol. \& Metab. 77:27-32.

18. Yki-Jarvinen, H., H. Vuorinen-Markkola, L. Koranyi, R. Bourey, K. Tordjman, M. Mueckler, A.M. Permutt, and V. Koivisto. 1992. Defect in insulin action on expression of the skeletal muscle/adipose tissue glucose transporter gene in skeletal muscle of type 1 diabetic patients. J. Clin. Endocrinol. \& Metab. 75:795-799.

19. Lund, S., H. Vestergaard, P.H. Andersen, O. Schmitz, L.B.H. Gotzsche, and O. Pedersen. 1993. GLUT4 content in plasma membrane of muscle from patients with non-insulin-dependent diabetes mellitus. Am. J. Physiol. 265: E889-E897.

20. Kahn, B.B. 1992. Facilitative glucose transporters: regulatory mechanisms and dysregulation in diabetes. J. Clin. Invest. 89:1367-1374.

21. King, P.A., M.F. Hirshman, E.D. Horton, and E.S. Horton. 1989. Glucose transport in skeletal muscle plasma membrane vesicles from control and exercise rats. Am. J. Physiol. 257:C1128-C1134.

22. Kahn, B.B., I.A. Simpson, and S.W. Cushman. 1988. Divergent mechanisms for the insulin resistant and hyperresponsive glucose transport in adipose cells from fasted and refed rats. J. Clin Invest. 82:691-699.

23. Kahn, B.B., and S.W. Cushman. 1987. Mechanism for markedly hyperresponsive insulin-stimulated glucose transport activity in adipose cells from insulin-treated streptozotocin diabetic rats. J. Biol. Chem. 262:5118-5124.

24. Clancy, B., and M. Czech. 1990. Hexose transport stimulation and membrane redistribution of glucose transporter isoforms in response to cholera toxin, dibutyryl cyclic AMP, and insulin in 3T3-L1 adipocytes. J. Biol. Chem. 265:12434-12443.

25. Clancy, B., S. Harrison, J. Buxton, and M. Czech. 1991. Protein synthesis inhibitors activate glucose transport without increasing plasma membrane glucose transporters in 3T3-L1 adipocytes. J. Biol. Chem. 266:10122-10130.

26. Harrison, S., J. Buxton, B. Clancy, and M. Czech. 1991. Evidence that erythroid-type transporter intrinsic activity is modulated by cadmium treatment of mouse 3T3-L1 cells. J. Biol. Chem. 266:19438-19449.

27. Harrison, S., J. Buxton, and M. Czech. 1991. Suppressed intrinsic catalytic activity of GLUT1 glucose transporters in insulin sensitive 3T3-L1 adipocytes. Proc. Natl. Acad. Sci. USA. 88:7839-7843.

28. Napoli, R., M.F. Hirshman, and E.S. Horton. 1995. Mechanisms and time course of impaired skeletal muscle glucose transport activity in streptozocin diabetic rats. J. Clin. Invest. 96:427-437.

29. Del Prato, S., R. Nosadini, A. Tiengo, P. Tessari, A. Avogaro, R. Trevisan, A. Valerio, M. Muggeo, C. Cobelli, and G. Toffolo. 1983. Insulin-mediated glucose disposal in type 1 diabetes: evidence for insulin resistance. J. Clin. Endocrinol. \& Metab. 57:904-910.

30. Yki-Jarvinen, H., and V.A. Koivisto. 1984. Continuous subcutaneous insulin infusion therapy decreases insulin resistance in type 1 diabetes. J. Clin. Endocrinol. \& Metab. 58:659-666.

31. Simonson, D.C., W.V. Tamborlane, R.S. Sherwin, J.D. Smith, and R.A. DeFronzo. 1985. Improved insulin sensitivity in patients with type 1 diabetes mellitus after CSII. Diabetes. 34[Suppl. 3]:80-86.

32. Lisato, G., I. Cusin, A. Tiengo, S. Del Prato, and B. Jeanrenaud. 1992. The contribution of hyperglycaemia and hypoinsulinaemia to the insulin resistance of streptozotocin-diabetic rats. Diabetologia. 35:310-315.

33. Barnard, R.J., J.F. Youngren, D.S. Kartel, and D.A. Martin. 1989. Effects of streptozotocin-induced diabetes on glucose transport in skeletal muscle. Endocrinology. 126:1921-1926.

34. Rizza, R.A., L.J. Mandarino, J. Genest, B.A. Baker, and J.E. Gerich. 1985. Production of insulin resistance by hyperinsulinemia in man. Diabetologia. 28:70-75.

35. Marangou, A.G., K.M. Weber, R.C. Boston, P.M. Aitken, J.C. Heggie, R.L. Kirsner, J.D. Best, and F.P. Alford. 1986. Metabolic consequences of prolonged hyperinsulinemia in humans. Evidence for induction of insulin insensi- tivity. Diabetes. 35:1383-1389.

36. Del Prato, S., F. Leonetti, D.C. Simonson, P. Sheehan, M. Matsuda, and R.A. DeFronzo. 1994. Effect of sustained physiologic hyperinsulinemia and hyperglycemia on insulin secretion and insulin sensitivity in man. Diabetologia. 37:1025-1035

37. Homan, M., C.P. Nicholson, H. Katz, J. Perkins, M. Haymond, M. Jensen, P. Butler, and R. Rizza. 1991. Effects of chronic systemic insulin delivery on insulin action in dogs. Diabetologia. 34:702-708.

38. Davalli, A.M., Y. Ogawa, L. Scaglia, Y.J. Wu, J. Hollister, S. BonnerWeir, and G.C. Weir. 1995. Function, mass, and replication of porcine and rat islets transplanted into diabetic nude mice. Diabetes. 44:104-111.

39. Bradford, M.M. 1976. A rapid and sensitive method for the quantitation of microgram quantities of protein, utilizing the principle of protein-dye binding. Anal. Biochem. 72:248-254.

40. Bers, D.M. 1979. Isolation and characterization of cardiac sarcolemma Biochim. Biophys. Acta. 555:131-146.

41. Bers, D.M., K.D. Philipson, and A.Y. Nishimoto. 1980. Sodium-calcium exchange and sideness of isolated cardiac sarcolemmal vesicles. Biochim. Biophys. Acta. 601:358-371.

42. Wilkinson, G.N. 1961. Statistical estimations in enzyme kinetics. Biochem. J. 80:324-332.

43. Wardzala, L.J., S.W. Cushman, and L.B. Salans. 1978. Mechanism of insulin action on glucose transport in the isolated rat adipose cell. J. Biol. Chem. 260:2197-2201.

44. Laemmli, U.K. 1970. Cleavage of structural proteins during the assembly of the head of bacteriophage $\mathrm{T}_{4}$. Nature (Lond.). 227:680-685.

45. Towbin, H., T. Staehelin, and J. Gordon. 1974. Electrophoretic transfer of proteins from polyacrylamide gels to nitrocellulose sheets: procedure and some applications. Proc. Natl. Acad. Sci. USA. 76:4350-4354.

46. Haspel, H.C., M.G. Rosenfeld, and O.M. Rosen. 1988. Characterization of antisera to a synthetic carboxyl-terminal peptide of the glucose transporter. J. Biol. Chem. 263:398-403.

47. James, D.E., K.M. Burleigh, and E.W. Kraegen. 1986. In vivo glucose metabolism in individual tissues of the rat. Interaction between epinephrine and insulin. J. Biol. Chem. 261:6366-6374.

48. Capaldo, B., R. Napoli, L. Di Marino, and L. Sacca'. 1992. Epinephrine directly antagonizes insulin-mediated activation of glucose uptake and inhibition of free fatty acid release in forearm tissue. Metab. Clin. Exp. 41:1146-1149.

49. Kubo, K., and J.E. Foley. 1986. Rate limiting steps for insulin-mediated glucose uptake into perfused rat hindlimb. Am. J. Physiol. 250:E100-E102.

50. Fink, R.I., P. Wallace, G. Brechtel, and J.M. Olefsky. 1992. Evidence that glucose transport is rate-limiting for in vivo glucose uptake. Metab. Clin. Exp. 41:897-902.

51. Katz, A., B.L. Nyomba, and C. Bogardus. 1988. No accumulation of glucose in human skeletal muscle during euglycemic hyper-insulinemia. Am. J. Physiol. 255:E942-E945.

52. Ziel, F.H., N. Venkatesan, and M.B. Davidson. 1988. Glucose transport is rate limiting for skeletal muscle glucose metabolism in normal and streptozocin-induced diabetic rats. Diabetes. 37:885-890.

53. Yki-Jarvinen, H., K. Sahlin, J.M. Ren, and V.A. Koivisto. 1990. Localization of rate-limiting defect for glucose disposal in skeletal muscle of insulin resistance type I diabetic patients. Diabetes. 39:157-167.

54. Luzi, L., A. Secchi, F. Facchini, A. Battezzati, C. Staudacher, D. Spotti, R. Castoldi, G. Ferrari, V. Di Carlo, and G. Pozza. 1990. Reduction of insulin resistance by combined kidney-pancreas transplantation in type 1 (insulindependent) diabetic patients. Diabetologia. 33:549-556.

55. Elahi, D., M. McAloon-Dyke, B.A. Clark, B.B. Kahn, J.E. Weinreb, K.L. Minaker, G.A. Wong, L.A. Morse, R.S. Brown, M.E. Shapiro, et al. 1993. Sequential evaluation of islet cell responses to glucose in the transplanted pancreas in humans. Am. J. Surg. 165:15-22.

56. Slot, J.W., H.J. Geuze, S. Gigengack, D.E. James, and G.E. Lienhard 1991. Translocation of the glucose transporter GLUT4 in cardiac myocytes of the rat. Proc. Natl. Acad. Sci. USA. 88:7815-7819.

57. Slot, J.W., H.J. Geuze, S. Gigengack, G.E. Lienhard, and D.E. James 1991. Immuno-localization of the insulin regulatable glucose transporter in brown adipose tissue of the rat. J. Cell Biol. 113:123-135.

58. Yang, J., and G.D. Holman. 1993. Comparison of GLUT4 and GLUT1 subcellular trafficking in basal and insulin-stimulated 3T3-L1 cells. J. Biol. Chem. 268:4600-4603.

59. Mueckler, M. 1993. The molecular biology of glucose transport: relevance to insulin resistance and non-insulin-dependent diabetes mellitus. J. Diabetes Complications. 7:130-141.

60. Satoh, S., H. Nashimura, A.E. Clark, I.J. Kozka, S.J. Vannucci, I.A Simpson, M.J. Quon, S.W. Cushman, and G.D. Holman. 1993. Use of bismannose photolabel to elucidate insulin-regulated GLUT4 subcellular trafficking kinetics in rat adipose cells. J. Biol. Chem. 268:17820-17829.

61. Holman, G.D., and S.W. Cushman. 1994. Subcellular localization and trafficking of the GLUT4 glucose transporter isoform in insulin-responsive cells. BioEssays. 16:753-759.

62. Klip, A. A. Marette, D. Dimitrakoudis, T. Ramlal, A. Giacca, Z. Q. Shi, and M. Vranic. 1992. Effect of diabetes on glucoregulation. Diabetes Care. 15: 1747-1766. 
63. Sumida, Y., K. Tsuchihashi, Y. Hotta, M. Yamada, A. Suzuki, A. Katsuki, K. Ito, M. Fujii, K. Murata, K. Nakatani, et al. 1995. Insulin action on the internalization of GLUT4 in isolated type II diabetic rat adipocytes. Diabetologia. 38[Suppl. 1]:A9. (Abstr.)

64. Giorgino, F., J.H. Chen, and R.J. Smith. 1992. Changes in tyrosine phosphorylation of insulin receptors and a 170,000 molecular weight nonreceptor protein in vivo in skeletal muscle of streptozotocin-induced diabetic rats: effects of insulin and glucose. Endocrinology. 130:1433-1444.

65. Folli, F., J.A. Saad, J.M. Backer, and C.R. Kahn. 1993. Regulation of phosphatidylinositol 3-kinase activity in liver and muscle of animal models of insulin-resistant and insulin-deficient diabetes mellitus. J. Clin. Invest. 92:17871794.
66. Dimitrakoudis, D., T. Ramlal, S. Rastogi, M. Vranic, and A. Klip. 1992. Glycaemia regulates the glucose transporter number in the plasma membrane of rat skeletal muscle. Biochem. J. 284:341-348.

67. Klip, A., T. Ramlal, D.A. Young, and J.O. Holloszy. 1987. Insulininduced translocation of glucose transporters in rat hindlimb muscles. FEBS Lett. 224:224-230.

68. Junod, A., A.E. Lambert, W. Stauffacher, and A.E. Renold. 1969. Diabetogenic action of streptozotocin: relationship of dose to metabolic response. J. Clin. Invest. 48:2129-2139.

69. Veleminsky, J., I.M. Burr, and W. Stauffacher. 1970. Comparative study of early metabolic events resulting from the administration of the two diabetogenic agents alloxan and streptozotocin. Eur. J. Clin. Invest. 1:104-108. 